\title{
Screening for cellulolytic plant enzymes using colorimetric and fluorescence methods
}

\section{Kirsten Krause and Stian Olsen}

Department of Arctic and Marine Biology, Faculty of Biosciences, Fisheries and Economics, UiT The Arctic University of Norway, Dramsvegen 201, 9037 Tromsø, Norway

\begin{abstract}
Cellulolytic activity can be measured using a variety of methods, the choice of which depends on the raw material and goals. An inexpensive, rapid and reliable method, suitable for plants and other sources alike, is based on digestion of the easily degradable soluble cellulose derivative carboxymethylcellulose (CMC). Direct detection of CMC digestion by cellulolytic activity is based on the "negative staining principle", where undigested CMC is stained with appropriate colorimetric or fluorescent stains, while CMC exposed to digestion by cellulase shows a reduction in staining intensity. The reduction is proportional to the enzyme activity and is not influenced by endogenous levels of glucose in the sample, making this method applicable for a wide variety of samples, including plant material.
\end{abstract}

\section{Keywords}

Calcofluor White Stain, Carboxymethylcellulose, cellulase, cellulose, Gram's Iodine Stain

\section{Introduction}

The plant cell wall component cellulose (ß-1,4-glucan) is among the most abundant biopolymers on earth. Its monomers, glucose molecules, are joined to form long linear chains 
that in turn use hydrogen bonds to align to stiff microfibrils that exhibit a high tensile strength. In plants these microfibrils confer strength and rigidity, providing a counter-force for the cell turgor. Enzymes that can break this rigid structure are found in plants [1] as well as in organisms feeding on living or dead plant material (e.g. [2-4]). In microbes, fungi and parasitic plants, cellulolytic enzymes facilitate plant infection.

The breakdown of cellulose is cooperatively mediated by endocellulases that randomly cleave internal bonds in the cellulose chains and by exocellulases that progressively cleave sugar units from the ends of the chains. Several methods have been developed for estimating the total saccharifying activity of crude cellulolytic extracts, mostly for the purpose of characterizing the capacity of soil-borne microorganisms to decompose cellulosic matter [46]. The most popular ones can be roughly divided into two types: qualitative petri dish-based assays and quantitative assays. In both cases, cellulosic substrates that are more readily soluble in water than pure cellulose, e.g. carboxymethylcellulose (CMC), have become the substrate of choice. In quantitative assays, the release of reducing sugars that can be monitored using spectrophotometric, fluorometric or chromatographic methods is used as a measure for cellulolytic activity. While this is a very valuable method for purified enzymes or partially purified extracts, it is prone to produce artifacts when sugars are naturally present at higher levels (such as in crude plant extracts).

In petri dish assays, the detection of the cellulolytic activity is achieved by staining of undigested CMC in areas not subjected to cellulose decomposition [7]. Typically, agar is used as an inert carrier substance into which the substrate is mixed and the staining is performed using Gram's Iodine stain, Safranin or Congo Red [8]. The visual results of CMC cleavage are clear halos surrounding the source of the enzyme [9], making this in theory a rapid, visual and inexpensive method. However, Zitomer and Eveleigh in 1987 [10] and more recently Johnsen and Krause [11] have advocated caution in the use of this method in connection with Gram's 
Iodine stain, as commercial agars were shown to produce artifacts with this dye. An even simpler method omitting the agar and exploiting the natural gelling capacity of the CMC substrate proved to be a good alternative. Moreover, when 96-well plates are used, a larger number of samples can be processed simultaneously with a minimal use of reagents [11]. Limitations, unfortunately, were still observed with crude plant extracts due to the interference with the spectrophotometric quantification by photosynthetic pigments and other extract discolorations. We have therefore tested a fluorescent dye as an alternative and found that this allows measuring crude plant extracts with little or no interference of endogenous sample components. This staining alternative is described alongside the colorimetric stain-based method that was published recently [11].

\section{Materials}

In the following, the materials needed for one 96-well plate assay are given.

\subsection{CMC solution}

Carboxymethylcellulose is commercially available in different viscosity grades. We use medium viscositiy CMC (e.g. Sigma-Aldrich, Catalog number C4888) at a concentration of 7\% in demineralized water, at which it has the consistency of very thick syrup and is difficult to pipet.

1. CMC powder, medium viscosity grade

2. demineralized water

3. Microwave oven

4. Duran glass bottle, magnetic stirrer and magnetic bar

\subsection{Colorimetric and fluorescent dyes}


1. For colorimetric detection: $20 \mathrm{ml}$ Gram's Iodine Stain $(0.133 \mathrm{~g} \mathrm{KI}$ and $0.067 \mathrm{~g}$ Iodine dissolved in $20 \mathrm{ml}$ demineralized water).

2. For fluorescence detection: $1 \mathrm{ml}$ Calcofluor White (CFW) Stain (Sigma-Aldrich, Catalog number 18909) composed of 1g/l Calcofluor White M2R (alias Fluorescent Brightener 28) and $0.5 \mathrm{~g} / \mathrm{l}$ Evans Blue.

\subsection{Cellulase calibration solution and extraction/dilution buffer}

Cellulase Onozuka R-10 from Trichoderma viride (Duchefa Catalog number C8001) at a stock concentration of $20 \mathrm{mg} / \mathrm{ml}$ in demineralized water. Dilutions of the stock for digestion were made in extraction/dilution buffer. The buffer is also used for preparing enzyme extracts from the samples.

Table 1: Extraction/dilution buffer

Chemical

Concentration

needed for $100 \mathrm{ml}$ buffer

\begin{tabular}{llc}
\hline Sodium ascorbate, $\mathrm{pH} 5.5$ & $50 \mathrm{mM}$ & $0.41 \mathrm{~g}$ \\
Sodium chloride & $300 \mathrm{mM}$ & $1.75 \mathrm{~g}$ \\
Sodium ascorbate (add fresh) & $20 \mathrm{mM}$ & $0.4 \mathrm{~g}$ \\
Calcium chloride & $10 \mathrm{mM}$ & $0.15 \mathrm{~g}$ \\
Glycerol, 85\% & $15 \%(\mathrm{v} / \mathrm{v})$ & $17.6 \mathrm{ml}$ \\
Polyvinylpyrrolidone (PVP-40) & $3 \%(\mathrm{w} / \mathrm{v})$ & $3 \mathrm{~g}$ \\
\hline
\end{tabular}

Chemicals were purchased from standard suppliers like Sigma, Merck, Fluka, etc. and are analytical grade.

\subsection{Miscellaneous equipment}

1. 96 well plates

2. Multipette (e.g. from Eppendorf) and matching Combitips for dispensing $200 \mu$ l volumes. 
3. Aluminum foil

4. Filter paper

5. $96 \%$ Ethanol

6. demineralized water

7. For fluorescence detection: Molecular Imager with UV-transillumination source for excitation and a 530/28 emission filter for blue fluorophore detection.

8. For colorimetric detection: plate reader for spectrophotometric absorbance measurements.

\section{Methods}

\subsection{Preparation of CMC solution}

1. Heat $25 \mathrm{ml}$ demineralized water in a $100 \mathrm{ml}$ Duran glass bottle briefly in a microwave oven without actually bringing it to boil.

2. Place the bottle on a magnetic stirrer and stir moderately with a magnetic bar.

3. Add $1.75 \mathrm{~g}$ of CMC powder slowly in small portions to the hot water, carefully avoiding clumping of the CMC.

The solution increases in viscosity as the concentration increases and as the solution cools down to room temperature. It should finally have a syrupy consistence, at which it can still be aspired and dispensed with a Multipette.

\subsection{CMC-CFW stain solution}

For fluorescence detection, the CFW stain can be added as soon as the CMC gel has cooled down to room temperature. Leave the bottle on the magnetic stirrer and add $1 \mathrm{ml}$ CFW stain to $25 \mathrm{ml} \mathrm{CMC}$. Wrap the bottle in aluminum foil and stir until the blue color (from Evans Blue) is evenly distributed. 


\subsection{Preparing a dilution series of cellulase for calibration}

1. Prepare stock solution by dissolving $20 \mathrm{mg}$ of cellulase Onozuka R10 (specific activity $>1000 \mathrm{U} / \mathrm{mg}$ ) in $1 \mathrm{ml}$ of sterile demineralized water.

2. Dilute $50 \mu \mathrm{l}$ stock solution 1:10 with $450 \mu \mathrm{l}$ extraction/dilution buffer.

3. Dilute $100 \mu$ l of the first dilution (above) 1:5 with $400 \mu$ l extraction/dilution buffer.

4. Dilute $100 \mu \mathrm{l}$ of the second dilution 1:5 with $400 \mu$ l extraction/dilution buffer.

5. Repeat dilutions in the same way until desired amount of dilution steps are reached.

\subsection{Sample extract preparation}

1. Grind $1 \mathrm{~g}$ of plant material using mortar and pestle and a pinch of sea sand in $1 \mathrm{ml}$ ice-cold extraction/dilution buffer.

2. Incubate for 2 hours on ice

3. Centrifuge the homogenate for 5 minutes at $12000 \mathrm{xg}$

4. Use the supernatant undiluted or diluted in extraction/dilution buffer for subsequent enzyme assays.

\subsection{Preparation of plates}

The steps below are schematically depicted in Figure 1. The colorimetric assay was described by Johnsen and Krause [11]. Figure 2 shows an example of a sample loading scheme.

1. Dispense $0.2 \mathrm{ml} \mathrm{CMC} \mathrm{solution} \mathrm{(for} \mathrm{colorimetric} \mathrm{detection)} \mathrm{or} \mathrm{CMC-CFW} \mathrm{solution} \mathrm{(for}$ fluorescence detection) into each well of a 96-well plate using a multipette dispenser.

2. For fluorescence detection only: Record fluorescence levels in each well before adding the samples (Fig. 3a).

3. Freshly prepare $0.1 \mathrm{ml}$ of each sample or enzyme for the calibration curve in extraction/dilution buffer and carefully overlay the CMC/CMC-CFW with it. 
4. Incubate for 16 hours in the dark at $27^{\circ} \mathrm{C}$.

\subsection{Differentiation of staining and detection}

\subsubsection{Colorimetric detection}

1. Remove enzyme solutions or samples by inverting the plate onto a piece of filter paper and lightly tapping it.

2. Add $0.2 \mathrm{ml}$ of Gram's Iodine Stain and stain for 2 hours in the dark at $27^{\circ} \mathrm{C}$. Remove stain as described in 1.

3. Differentiate staining by carefully adding $0.2 \mathrm{ml} 96 \%$ Ethanol to each well and removing it after 5 minutes as described in 1.

4. Repeat step 3.

5. Repeat step 4, but wait for 15 minutes before removing the ethanol.

6. Record the absorbance at $575 \mathrm{~nm}$

7. Calculate enzyme activity using the calibration curve (see examples shown in Fig. 3c and d) or select extracts of interest based on visual inspection of the results.

\subsubsection{Fluorescence detection}

1. Remove enzyme solutions or samples by inverting plate onto a piece of filter paper and lightly tapping it.

2. Differentiate staining by carefully adding $0.2 \mathrm{ml}$ demineralized water to each well and removing it after 15 minutes as described in 1.

3. Repeat step 2, but wait for 30 minutes before removing the water as described in 1 .

4. Repeat step 2, but wait for 90 minutes before removing the water as described in 1 .

5. Record staining intensity using a Molecular Imager. Excitation of fluorescence is carried out using UV light (UV table) and emission is recorded at $530 \mathrm{~nm}$ (Fig. 3b).

6. Calculate enzyme activity using the calibration curve (see examples shown in Fig. 3c and d) or select extracts of interest based on visual inspection of the results. 


\section{Notes}

1. Unused extracts and ascorbate-containing buffer can be stored at $-20{ }^{\circ} \mathrm{C}$ but may loose activity if freeze-thawed frequently or stored over a longer period.

2. For negative controls, enzyme extracts can be inactivated by heating to $95^{\circ} \mathrm{C}$.

3. Recording of the fluorescence before incubation can be omitted if sample readings are related to control wells without enzyme. However, this step gives the possibility to correct for a priori CMC volume differences due to pipetting errors. Given the gelatinous nature of the CMC solution, such differences are difficult to avoid completely.

3. The outermost rows and columns of the 96-well plates show strong reflection during illumination on a UV table. (Fig. 4b) and should not be used for samples or calibration curves. 4. Bubbles in the wells (Fig. 4b) show reflection and can interfere with measuring accuracy. They should thus be avoided. 


\section{Acknowledgements}

The authors thank Tromsø Research Foundation (Mohn Foundation) and the Faculty of Biosciences, Fisheries and Economics at UiT for providing the funds for this work. Marie Cooper (UiT) is thanked for proof-reading.

\section{Reference list}

1. Popper, Z.A., et al., Evolution and diversity of plant cell walls: from algae to flowering plants. Annu Rev Plant Biol, 2011. 62: p. 567-90.

2. Kudo, T., Termite-microbe symbiotic system and its efficient degradation of lignocellulose. Bioscience, Biotechnology, and Biochemistry, 2009. 73(12): p. 2561-7.

3. Cai, S., et al., Cellulosilyticum ruminicola, a newly described rumen bacterium that possesses redundant fibrolytic-protein-encoding genes and degrades lignocellulose with multiple carbohydrate- borne fibrolytic enzymes. Appl Environ Microbiol, 2010. 76(12): p. 3818-24.

4. Dashtban, M., et al., Fungal biodegradation and enzymatic modification of lignin. Int J Biochem Mol Biol, 2010. 1(1): p. 36-50.

5. Vancov, T. and B. Keen, Rapid isolation and high-throughput determination of cellulase and laminarinase activity in soils. J Microbiol Methods, 2009. 79(2): p. 174-7.

6. Zhang, Y.H., J. Hong, and X. Ye, Cellulase assays. Methods in Molecular Biology, 2009. 581: p. 213-31.

7. Dashtban, M., H. Schraft, and W. Qin, Fungal bioconversion of lignocellulosic residues; opportunities \& perspectives. Int J Biol Sci, 2009. 5(6): p. 578-95.

8. Gohel, H.R., et al., A comparative study of various staining techniques for determination of extracellular cellulase activity on Carboxy Methyl Cellulose (CMC) agar plates. Int J Curr Microbiol and Appl Sci, 2014. 3(5): p. 261-266.

9. Kasana, R.C., et al., A rapid and easy method for the detection of microbial cellulases on agar plates using gram's iodine. Curr Microbiol, 2008. 57(5): p. 503-7.

10. Zitomer, S.W. and D.E. Eveleigh, Cellulase screening by iodine staining: An artefact. Enzyme and Microbial Technology, 1987. 9(4): p. 214-216.

11. Johnsen, H.R. and K. Krause, Cellulase activity screening using pure carboxymethylcellulose: application to soluble cellulolytic samples and to plant tissue prints. Int J Mol Sci, 2014. 15(1): p. 830-8. 


\section{Figure legends}

Figure 1: Flow diagram showing the key steps of the two alternative detection methods.

* : Protect from light. $\S$ : Optimal incubation temperature may vary with the type of sample.

Figure 2: Loading scheme example for the fluorescence assay method in 96-well plate format. The scheme shown here (a) allows for 8 samples (green fields, I to VIII) and two dilution series of the calibration enzyme (grey fields) to be compared. For each sample, two dilutions (e.g. Ia and Ib), and three technical replicates are loaded. Yellow fields are left empty because the outer rows and colums of each plate tend to show higher reflection when detecting fluorescence (see also Figure 4a)

Figure 3: Example for evaluation of CMC digestion by cellulolytic action. $(a, b)$ Fluorescence of CMC-CFW is detected before (a) and after (b) incubation with the samples. The labeling of rows (B-G) and columns (2-11) corresponds to the scheme shown in Figure 2. (c) A calibration curve was calculated from the difference in fluorescence values of wells containing defined amounts of cellulose before and after incubation with the enzyme (squares with broken blue-white lines in (b)). The difference increases with increasing units of cellulase. The linear range of activity detection is between 0 and $\sim 100$ milliunits (mU) enzyme. (d) The difference in fluorescence values before and after incubation is plotted for 48 data points (outlined in yellow in (b)), corresponding to eight samples, each in two dilutions and in technical triplicates (d). Filled symbols represent undiluted samples, open symbols represent the same samples after a 1:5 dilution. 
Figure 4: Potential caveats. (a) Heat inactivation at $95^{\circ} \mathrm{C}$ for 15 minutes is effective in the linear range of enzyme activity detection, between 0 and 100 milliunits (see Fig. 3). (b) Reflection in the outer rows and colums of each plate (upper arrow) and from air bubbles (lower arrow) in the CMC matrix can give false results when detecting fluorescence. 


\section{Colorimetric Detection:}
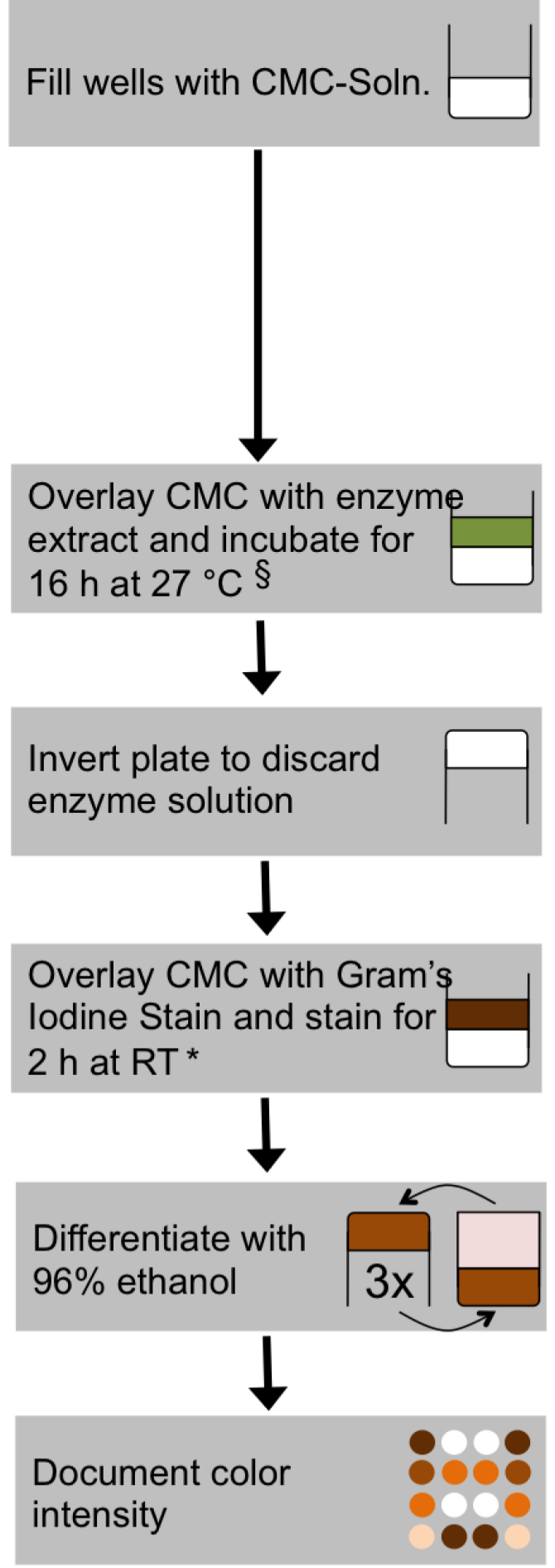

\section{Fluorescent Detection:}

Fill wells with CMC-Calcofluor White Stain-Soln. *

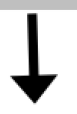

Document fluorescence intensity

Overlay CMC with enzyme extract and incubate for $16 \mathrm{~h}$ at $27^{\circ} \mathrm{C} *, \S$

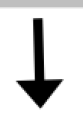

Invert plate to discard enzyme solution
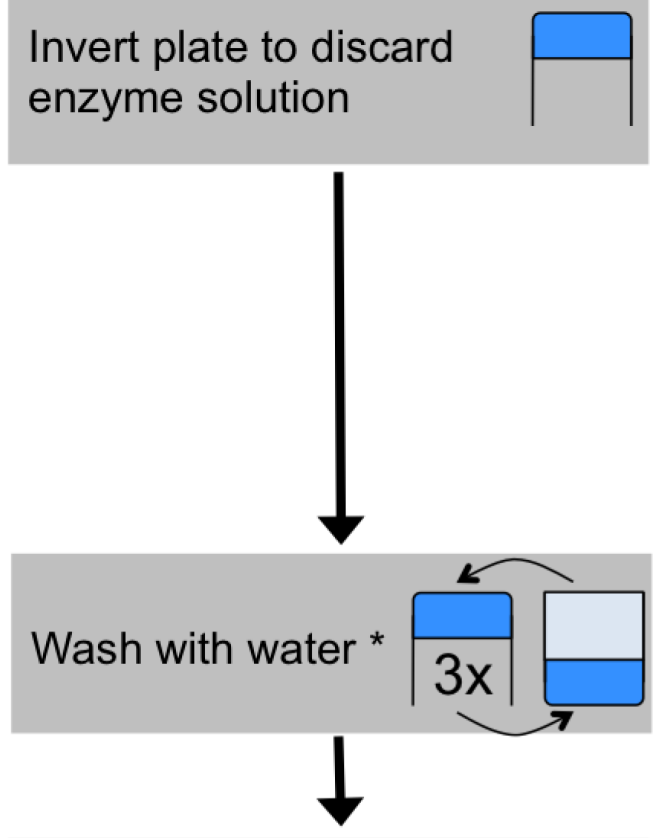

Document fluorescenc6. intensity 


\begin{tabular}{|c|c|c|c|c|c|c|c|c|c|c|c|c|c|}
\hline & 1 & 2 & 3 & 4 & 5 & 6 & 7 & 8 & 9 & 10 & 11 & 12 & \\
\hline A & empty & empty & empty & empty & empty & empty & empty & empty & empty & empty & empty & empty & A \\
\hline B & empty & $0.64 \mathrm{mU}$ & Ia & IIIa & $\mathrm{Va}$ & VIIa & Ib & IIIb & $\mathrm{Vb}$ & VIIb & $2000 \mathrm{mU}$ & empty & B \\
\hline C & empty & $3.2 \mathrm{mU}$ & Ia & IIIa & $\mathrm{Va}$ & VIIa & Ib & IIIb & $\mathrm{Vb}$ & VIIb & $400 \mathrm{mU}$ & empty & C \\
\hline D & empty & $16 \mathrm{mU}$ & Ia & IIIa & $\mathrm{Va}$ & VIIa & Ib & IIIb & $\mathrm{Vb}$ & VIIb & $80 \mathrm{mU}$ & empty & D \\
\hline E & empty & $80 \mathrm{mU}$ & IIa & IVa & VIa & VIIIa & IIb & IVb & VIb & VIIIb & $16 \mathrm{mU}$ & empty & $\mathbf{E}$ \\
\hline $\mathbf{F}$ & empty & $400 \mathrm{mU}$ & IIa & IVa & VIa & VIIIa & IIb & $\mathrm{IVb}$ & VIb & VIIIb & $3.2 \mathrm{mU}$ & empty & $\mathbf{F}$ \\
\hline G & empty & $2000 \mathrm{mU}$ & IIa & IVa & VIa & VIIIa & IIb & IVb & VIb & VIIIb & $0.64 \mathrm{mU}$ & empty & G \\
\hline \multirow[t]{2}{*}{ H } & empty & empty & empty & empty & empty & empty & empty & empty & empty & empty & empty & empty & H \\
\hline & 1 & 2 & 3 & 4 & 5 & 6 & 7 & 8 & 9 & 10 & 11 & 12 & \\
\hline
\end{tabular}

Figure 2 
a

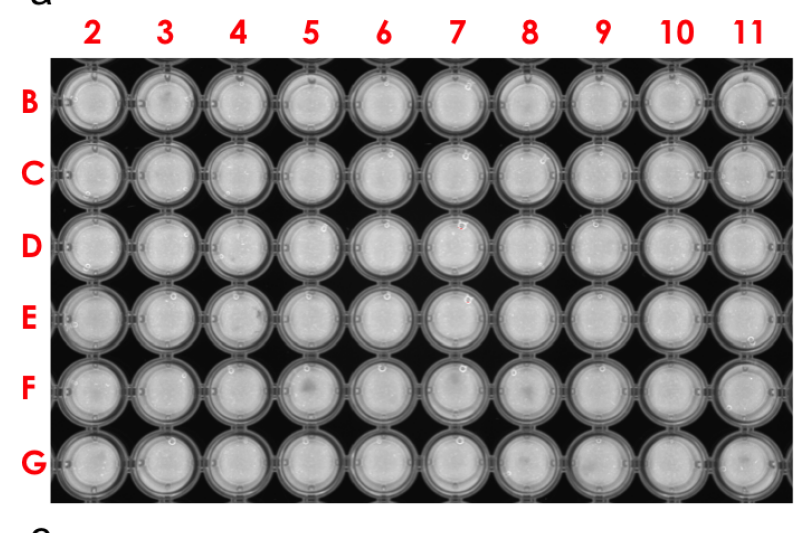

C

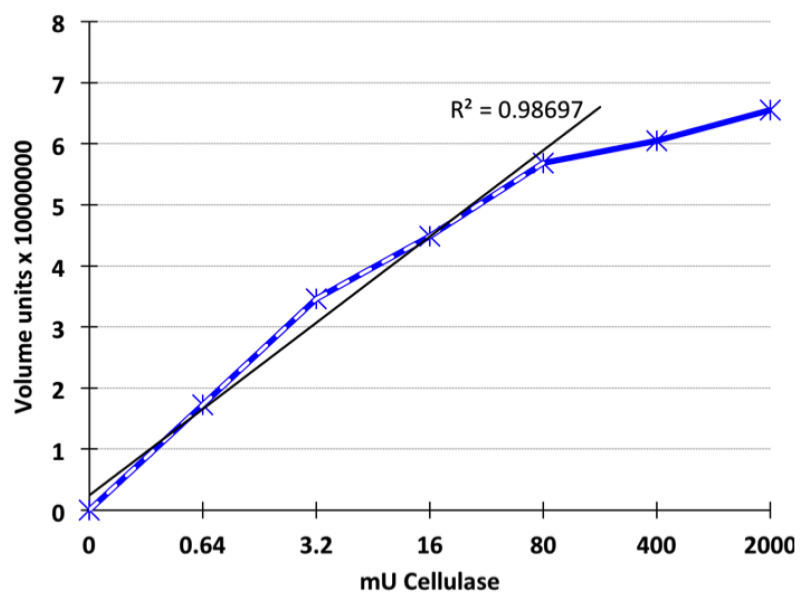

b

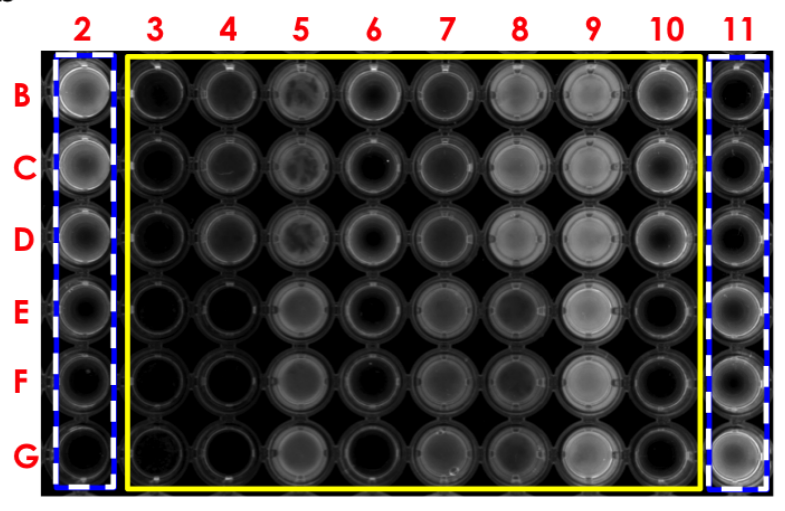

d

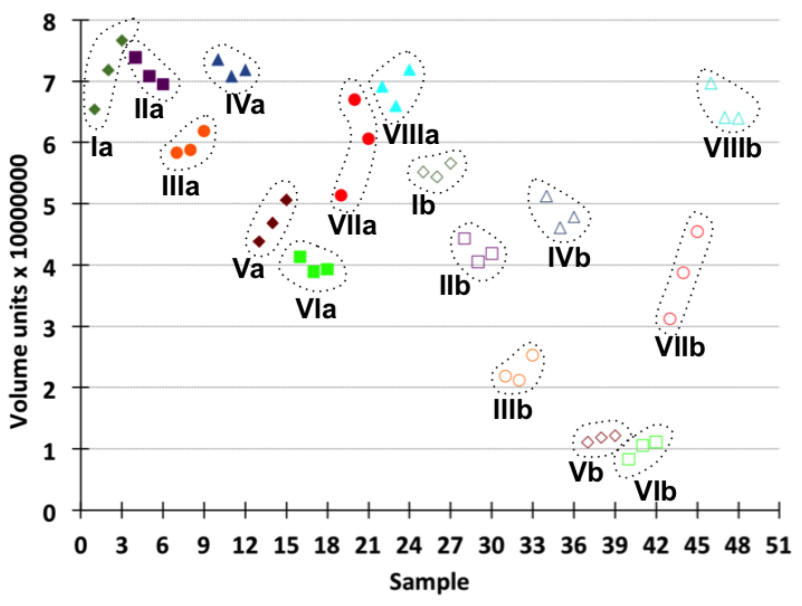

Figure 3 


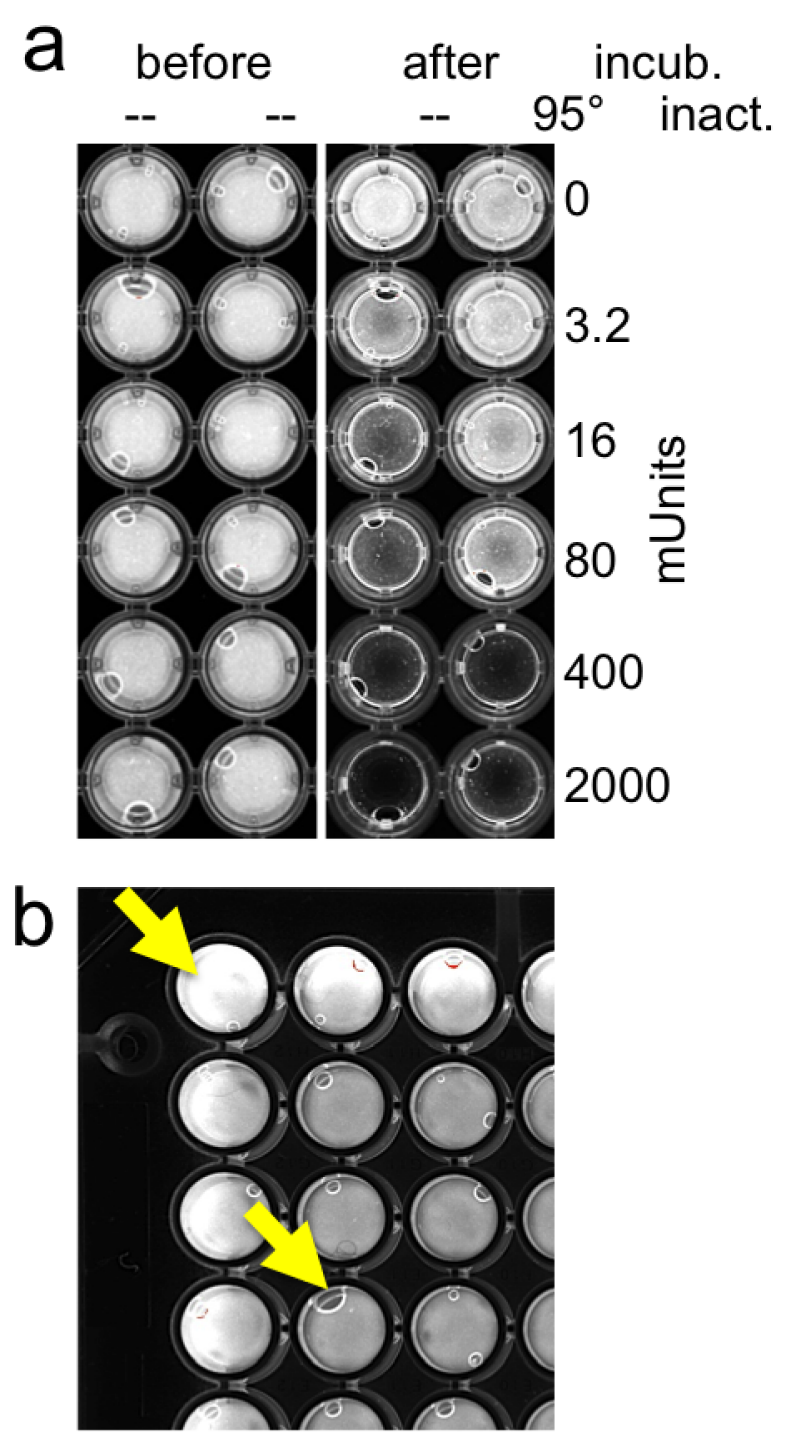

Figure 4 